\title{
Analysis on Risks and Hazards of the Sloshing Liquid Cargo Tank in Oil Tanker
}

\author{
Zhu Fa-xin \\ School of Maritime and Civil Engineering of ZJOU \\ ZheJiang Ocean University \\ ZhouShan, China \\ zhufax7758@163.com
}

Lu Jin-shu

School of Maritime and Civil Engineering of ZJOU

ZheJiang Ocean University

ZhouShan, China

goldtree_lu@163.com

\author{
Li Yu-le \\ School of Maritime and Civil Engineering of ZJOU \\ ZheJiang Ocean University \\ ZhouShan, China \\ 704952772@qq.com \\ Gan Xue-zhen \\ School of Maritime and Civil Engineering of ZJOU \\ ZheJiang Ocean University \\ ZhouShan, China \\ 50009283@qq.com
}

\begin{abstract}
Plenty of volatile organic compounds, inert gases, oxygen, carbon dioxide and other gases which are called as gas mixtures exist and mix in the upper space of liquid cargo tank owing to sloshing of oil tanker and heating of the crude oil in the liquid cargo tank. There will be potential risks of fires or explosions, air pollution and personnel poisoning in the oil tanker when gas mixtures escape from the liquid cargo tank and diffuse somewhere in oil tanker unusually. At the same time, potential risks of fires, explosions, collisions, groundings and oil spills et al. will also take place when operation of the tanker or work of the liquid cargo tank is carried out wrongly etc.. Once these potential risks are controlled improperly, the serious accidents will occur, resulting in huge losses and damages to oil tanker. In consideration of these potential risks existed in oil tanker, methods of VOCs processing are summarized, the current method of VOCs processing in the sloshing liquid cargo tank and its problems or deficiencies are analysed, the system diagram of the sloshing liquid cargo tank including 13 basic elements is established based on the theory of Man-Machine-Environment-Management System Engineering. Escaping and diffusion of the gas mixtures, VOCs fires and explosions, air pollution, personnel poisoning, collisions or groundings of oil tanker and oil spills of the main risks in the sloshing liquid cargo tank in ship are concluded from operation processes of loading, unloading and washing of crude oil in the system of the sloshing liquid cargo tank in oil tanker. What's more, the formation process of these risks and hazards caused by risks are analysed too.
\end{abstract}

Keywords-oil tanker; the sloshing liquid cargo tank; volatile organic compounds; risk; risk hazard.

\section{INTRODUCTION}

At present, oil tanker accounts for approximately 35\% in the total quantity of the ships, which plays a very important role in the world's shipping industry. With the development of the world economy and the strengthening of the international trade, the quantity of crude oil tanker and product carrier will continue to increase at a higher level ${ }^{[1]}$. However, oil tanker has its own characteristics, in which bigger potential risks exist when oil tanker is loaded with thousands or more of tons of crude oil. As we know, most oil is a flammable substance of the viscous liquid or semisolid which is comprised of various hydrocarbons. Owing to the sloshing of the tanker and the need of the oil heating etc., VOCs will speed up to volatilize. There will be potential risks of VOCs fires or explosions, air pollution and personnel poisoning when gas mixtures containing VOCs, inert gases, oxygen, carbon dioxide and other gases escape and diffuse unusually. At the same time, the potential risks of VOCs fires or explosions, air pollution, personnel poisoning, collisions, groundings, oilspills or other accidents will also take place when operations of the tanker are carried out wrongly. Once these potential risks are controlled improperly, the serious accidents will occur, resulting in the huge losses and damages to the oil tanker.

According to statistics from ITOPF(the international tanker owners pollution federation limited) by January 2015, about 10000 accidents of collisions, groundings, fires and explosions in oil tankers have taken place since 1970, resulting in serious leakages, casualties and pollution ${ }^{[2]}$. Although these accidents have decreased significantly in recent years, risks and hazards caused still can't be ignored because of more and more bigger tonnage of oil tanker. Therefore, it is very important to analyse the risks and their hazards of the sloshing liquid cargo tank in oil tanker.

\section{THE CURRENT METHOD AND ITS PROBLEMS OF VOCS PROCESSING IN OIL TANKER}

In the sloshing liquid cargo tank, there are uneven multiphysics fields of concentration, temperature, pressure and density because of sloshing of oil tanker, heating of the crude oil and uneven distribution of the pipework and so on, in which concentration, temperature and pressure of the VOCs, and oxygen and other physical parameters need to draw attention carefully. Some accidents of fires, explosions, air pollution or personnel poisoning etc. will take place when gas mixtures escape from the liquid cargo tank and diffuse 
somewhere in oil tanker unusually, endangering safety of oil tanker and personnel and resulting in huge losses ${ }^{[3-4]}$.

\section{A. Common methods of VOCs processing}

VOCs processing methods are as follows, absorption, adsorption, condensation, membrane separation, and catalytic oxidation-combustion $\operatorname{method}^{[5-9]}$, or a comprehensive method of two or more methods mentioned above. these methods are briefly introduced below.

1) Absorption method

At certain temperature and pressure, the absorbent is used to absorb some hydrocarbon components in gas mixtures rather than other components, then absorbed hydrocarbon components are resolved to recycle and reuse VOCs. The method is divided into two methods by values of pressure and temperature.

\section{2) Adsorption method}

The adsorbent or activated carbon is used to adsorb some hydrocarbon components rather than other components based on different adsorption affinity between hydrocarbon components and other components in gas mixtures, then adsorbed hydrocarbon components are resolved to recycle and reuse VOCs. The adsorbent or adsorbent material will saturate quickly when the concentration of VOCs is at a very higher level, which raises strict demand for life cycle of adsorbent device, safety of the resolving process and so on.

\section{3) Condensation method}

In this method, condenser is used, in which refrigerant and gas mixtures exchange heat. Under usual atmospheric pressure, gas mixtures is cooled down to a lower temperature in order to recycle and reuse the liquid that most hydrocarbon components of gas mixtures were condensed to be, the other gas in gas mixtures is directly discharged into the atmosphere.

\section{4) Membrane separation method}

The VOCs is separated from gas mixtures based on different component gas in gas mixtures having different permeation rate under certain pressure.

\section{5) Catalytic oxidation method}

This method is operated simply, as its cost is lower, and combustion heat of VOCs is reused, but the question of the safety and environment need to be considered.

6) Comprehensive method

It is a comprehension of different methods, e.g. absorption method and catalytic oxidation-combustion method are used together. In this method, hydrocarbon components in gas mixtures are absorbed dispersedly, then are oxidated and combusted by use of the catalyzer, and its cost is equal almost to absorption method.

These methods mentioned above have been used well to oil truck, oil storage base, oil station and so on, reducing effectively air pollution and personnel poisoning, VOCs are recycled or combustion heat is utilized, but these methods are seldom used in oil ship.

\section{B. The current method and its problems of VOCs processing in oil tanker}

\section{1) The current method of VOCs processing}

When oil tanker is loading, unloading or sailing, VOCs will volatilize or evaporate continuously from liquid cargo tank owing to sloshing of oil tanker and heating of the oil and other causes. As concentration of VOCs continues to rise, fires or explosions might take place in liquid cargo tank in the case of fire resource, electric sparks and static sparks.
There will also be potential risks of personnel poisoning, air pollution or others when gas mixtures are directly discharged overboard without any processing. So it is necessary to process gas mixture by some method.

In oil tanker, the current method of VOCs processing is filling inert gases into oil tank and using breathing valves or gas holes of single direction. Relevant process is as follows.

In order to reduce concentration of VOCs in oil tank and avoid fires or explosions, some inert gases are filled into oil tank. As VOCs volatilize continuously, concentration and pressure of VOCs rise, breathing valves will open automatically, excessive gas mixtures are discharged overboard. Breathing valves will close automatically when pressure of gas mixtures in oil tank is lower. At the moment, concentration of VOCs is low enough, so the risks of fires and explosions drop, ensuring the safety of oil tanker and personnel.

2) Problems in current method of VOCs processing

Actually, the current method of VOCs processing in oil tanker by filling inert gases and using breathing valves is effective to avoid fires and explosions in oil tank, but it still has some problems. 1)Plenty of toxic gaseous hydrocarbons contained in VOCs lead to health problems of chronic poisoning, nausea and fatigue so on. 2)VOCs discharged overboard directly without any processing may form photochemical smog, causing secondary pollution of the atmosphere. 3)VOCs are light hydrocarbon components in crude oil, whose discharge affects combustion quality of the oil and leads to a waste of crude oil. 4)Fires and explosions may result from improper operations, broken breathing valves and other reasons.

\section{ANALYSIS ON RISKS OF THE SLOSHING LIQUID CARGO TANK IN OIL TANKER}

\section{A. The theory of Man-Machine-Environment-Management System Engineering}

In Man-Machine-Environment System Engineering (MMESE), the theory of system science and method of system engineering are applied to deal with the relationships of three factors among man, machine and environment correctly. MMESE is a science of optimal combination in MME system, whose object of study is Man-MachineEnvironment system ${ }^{[10]}$. With the gradual development of system science theory, important role of the factor of management in system is gradually recognized by the experts, so a more perfect theory of man-machineenvironment-management system engineering(MMEMSE) is formed.

In MMEMSE, man refers to main body of operating personnel, management personnel and so no. Machine relates to all objects controlled and operated by man, such as systems, equipments or devices in the production and operation processes. Environment involves given surrounding conditions that man and machine contact with, e.g. inside environment, outside environment and so on. Management is an objective rule among system elements. The optimal aims of the system is safety, efficiency and economy.

\section{B. The diagram of the sloshing liquid cargo tank system}

According to the theory of MMEMSE, the diagram of the sloshing liquid cargo tanker system was shown in Figure $1 .$. 


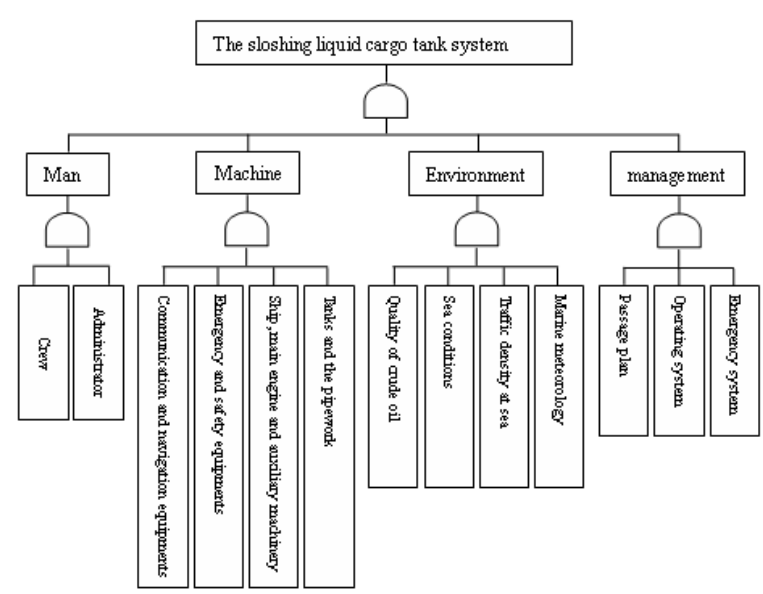

Figure 1. Diagram of the sloshing liquid cargo tank system

C. The functions of components in the sloshing liquid cargo tank system

A component of the sloshing liquid cargo tank system has its own function, which is shown in detail in Table 1. as follows.

Tab. 1 Functions of components in the sloshing liquid cargo tank system

\begin{tabular}{|c|c|c|}
\hline Number & Component & $\begin{array}{ll}\text { Function } \\
\end{array}$ \\
\hline 1 & Crew & $\begin{array}{l}\text { In charge of the operations and maintenance } \\
\text { work of oil tank. }\end{array}$ \\
\hline 2 & Administrator & $\begin{array}{l}\text { To provide the traffic service in sea, make } \\
\text { quick response to the traffic condition and give } \\
\text { the feedback to the ship by the radio. }\end{array}$ \\
\hline 3 & $\begin{array}{l}\text { Communicati } \\
\text { on and } \\
\text { navigation } \\
\text { equipments }\end{array}$ & $\begin{array}{l}\text { To provide communications between the ship } \\
\text { and ship, ship and administrator } \\
\text { department, display the ship’s position and its } \\
\text { direction. }\end{array}$ \\
\hline 4 & $\begin{array}{l}\text { Emergency } \\
\text { and safety } \\
\text { equipments }\end{array}$ & $\begin{array}{l}\text { Emergency generator and fire pump, air } \\
\text { compressor and other equipments determine } \\
\text { the emergency response capacity of the ship. } \\
\text { The safety equipments of lifeboat, raft, life } \\
\text { buoysetc. determine risk resistance capacity in } \\
\text { case of abandoning ship. }\end{array}$ \\
\hline 5 & $\begin{array}{l}\text { Ship, main } \\
\text { engine and } \\
\text { auxiliary } \\
\text { machinery }\end{array}$ & $\begin{array}{l}\text { The overall conditions of the ship's age, type } \\
\text { and others decide abilities of ship } \\
\text { performance and risk resistance, the main } \\
\text { engine and auxiliary machinery provide the } \\
\text { power and electric power required etc.. }\end{array}$ \\
\hline 6 & $\begin{array}{l}\text { Tanks and the } \\
\text { pipework }\end{array}$ & $\begin{array}{l}\text { Arrangements of the sloshing liquid cargo } \\
\text { tanks and their pipework determine the hazards } \\
\text { of risk of ship, affect the ability of risk } \\
\text { resistance of the ship. }\end{array}$ \\
\hline 7 & $\begin{array}{l}\text { Quality of } \\
\text { crude oil }\end{array}$ & $\begin{array}{l}\text { To determine gas components volatilized from } \\
\text { oil tanks, influence the safety and the degree of } \\
\text { environment pollution etc.. }\end{array}$ \\
\hline 8 & $\begin{array}{l}\text { Sea } \\
\text { conditions }\end{array}$ & $\begin{array}{l}\text { Sea conditions of the seawater density, float } \\
\text { bowl, buoys, geological features, and depth } \\
\text { determine the ship draught, ship operation, } \\
\text { may cause ground even. }\end{array}$ \\
\hline 9 & $\begin{array}{l}\text { Traffic } \\
\text { density at sea }\end{array}$ & $\begin{array}{l}\text { To determine the ship operations, and may } \\
\text { cause collisions of the ship etc.. }\end{array}$ \\
\hline 10 & $\begin{array}{l}\text { Marine } \\
\text { meteorology }\end{array}$ & $\begin{array}{l}\text { Marine meteorology of the } \\
\text { wind, wave, flow and other conditions decides } \\
\text { ship operation and safety etc.. }\end{array}$ \\
\hline 11 & Passage plan & To determine the potential risks of the ship. \\
\hline 12 & $\begin{array}{l}\text { Operating } \\
\text { system }\end{array}$ & To determine capacities of the ship operations. \\
\hline 13 & $\begin{array}{l}\text { Emergency } \\
\text { system }\end{array}$ & $\begin{array}{l}\text { To determine capacities of the risk elimination } \\
\text { and emergency escape on emergent occasion. }\end{array}$ \\
\hline
\end{tabular}

D. Operation processes of the sloshing liquid cargo tank system in oil tanker ${ }^{[11]}$

1) Operation process of cargo oil loading

a) Preparation stage of cargo oil loading
Check the connection of grounds, the connections between loading arms and cargo oil pipes, oil barrels or drums, sounding equipments of the oil level, valves, operation system of hydraulic oil power station, safety devices and alarm systems and inert gas system etc. are in normal working condition.

\section{b) The stage of cargo oil loading}

Chief officer or mate arranges for staff on duty, and open the main valve of the outlet duct when confirmation is finished by two sides of the ship and the shore. At the beginning, cargo oil is pumped at a low speed. After the cargo oil loading is normal, loading rate is increased by the shore side to rating speed. The quantity of the loading oil and the height of empty space in oil tank should be measured and recorded every two hours, the draft, freeboard and tidal condition etc. should also be checked.

\section{c) The final stage of cargo oil loading}

Near the final stage of cargo oil loading, the loading rate should be reduced as the shore side is noticed. When oil level of the a cargo tank is about to achieve the given height of empty space in oil tank, the valve of the next oil tank needed to trim should be opened, former valve should be closed then. Trimming operation of the tanks is over, high pressure air or nitrogen system can be started by the shore side, in other words, the operation of oil recovering can be started. When oil recovering operation is finished, pumping oil ducts should be dismantled. Finally, check that all valves of the liquid cargo tanks are in closed condition.

\section{2) Operation process of cargo oil unloading}

\section{a) Preparation stage of cargo oil unloading}

Both the ship side and the shore side discuss and confirm on information of the maximum rate of oil unloading, the highest pressure of the outlet duct, the procedure of the oil unloading, draught of the ship, the maximum degrees of transverse and longitudinal inclination etc.. All items in the checklist should be checked one by one, cargo oil reserved in oil tanks should also be measured.

\section{b) The stage of cargo oil unloading}

Check that the valves and pipelines of the centrifugal pump are in good order, cargo oil pump should be operated at a low speed at the beginning stage until the oil unloading system is in order, then the speed of oil unloading can be increased gradually. Near the final stage of cargo oil unloading, rotation speed or the outlet valve of the centrifugal pump should be cut down to reduce the displacement of the centrifugal pump to make sure the normal working of the centrifugal pump.

\section{c) The final stage of cargo oil unloading}

Near the final stage of cargo oil unloading, check that every working part of the stripping pump is in normal work. When the stripping pump is in operation, the steam valves should be closed cautiously, the drain cocks should be opened to drain residual water. After striping operation of the tanks is over, high pressure air or nitrogen system can be used by the ship side to strip cargo oil reserved in the loading arms or oil hoses to oil drums or oil barrels on the shore.

3) Crude oil washing process of liquid cargo tank

a) Preparation stage of crude oil washing

crude oil washing(COW) is expressed by the abbreviation of COW. Preparatory works, including confirming of the oxygen content kept in the tank, hanging of the billboards, checking of the safety etc. should be 
completed. Check the degrees of transverse and longitudinal inclination of the ship and the height of the oil level in tanks, and adjust to required values.

\section{b) The stage of COW}

Strip the tanks with oil injector, open the pump valves in the crude oil washing pipeline. Use cargo oil pump at a low speed to pump oil to washing pipeline or oil injector, open washing valves and adjust the speed to three quarters of the rating speed, keep the main valve of the washing machine on deck open, increase the pump speed, open slowly the main valve in washing branch pipe to exhaust the gases in washing pipelines. When the gases are exhausted, the main valve should be opened completely. In the process of washing operation, oil temperature, gas pressure and oxygen content in liquid cargo tank should be controlled, make sure that no leakages take place.

\section{c) The final stage of $\mathrm{COW}$}

When the washing machine turns $0^{\circ}$, firstly reduce the speed of the cargo oil pump, then close the main valve in the washing branch pipe, stop the washing machine, lastly, close the inlet and outlet valve in the driving unit of the washing machine. Confirm the quantity of residual oil, continue to strip the tanks a few minutes until the residual oil is drained completely, then stop the cargo oil pump. Strip residual oil in the pipelines to slop tank by stripping pump. Close the main valve on deck, open other washing valves and the main valve in the branch pipe on deck. A few minutes later, close all the valves above.

\section{E. Analysis on Risks of the sloshing liquid cargo tank}

In the navigation process of oil tanker loading lots of cargo oil, there will be potential risks of VOCs fires, explosions, diffusion of the gas mixtures, collisions, groundings and oil spills etc. owing to sloshing and improper operation of ship etc..

In the process of oil loading, unloading and COW, there will be potential risks of VOCs fires, explosions, escaping and diffusion of the gas mixtures, collisions, groundings and oil spills etc.

Therefore, it is concluded easily that there are potential risks of diffusion of the gas mixtures, VOCs fires, explosions, collisions, groundings and oil spills etc in the sloshing liquid cargo tank in oil tanker ${ }^{[12]}$.

\section{ANALYSIS ON HAZARDS OF RISKS IN THE SLOSHING} LIQUID CARGO TANK

\section{A. Risk hazards of gas mixtures diffusion}

In the processes of oil loading and unloading, gas mixtures escaped from breathing valves of the oil tank may be blown or diffused to living area, kitchen and engine room etc., and make these areas filled with combustible gases, cause fires of oil tanker. At the same time, the fires happening in one area may spread to another area by diffusion of the gas mixtures, and cause fires, leading to bigger harm.

The quality of cargo oil will be affected because of escaping and diffusion of the VOCs. VOCs escaped are light hydrocarbon components in cargo oil, which affect the combustion quality of the oil.

There are plenty of toxic gaseous hydrocarbons in VOCs, whose specific gravity is heavier than the air, floating above the ground, leading to bad effects on the crew and environment. If gas mixtures are inhaled by the crew, chronic poisoning will be caused, even the spiratory system and the central nervous system will be damaged in serious cases, causing breathing difficulties, dizziness, fatigue, nausea, vomiting and other hazards.

If VOCs are discharged overboard directly, there will not only be a direct damage to the atmosphere, but also form photochemical smog, which is one of the main pollutants in modern industrial society.

\section{B. Risk hazards of VOCs fire and explosion}

VOCs fire is a chemical change, while VOCs explosion is a physical change where gas pressure rises rapidly in the liquid cargo tank, there is some relation between VOCs fire and VOCs explosion.

When the concentration of VOCs and oxygen in the liquid cargo tank reaches a higher value, there is a flame, electric spark or static spark etc. at the same time, VOCs will be ignited, soon VOCs fire will be spread to the whole tank, and gas pressure in local tank rises rapidly. Due to firing gases restricted in the oil tank, gas pressure continues to rise until the bulkhead cracks, the initial explosion occurs. If remedial measures are improper, lots of air will get into oil tank. Once VOCs are supplied for more air, there will be a violent secondary explosion.

VOCs explosion is also a kind of fire spread, whose light and heat become a fire source of the adjacent tank, leading to fire and explosion in the adjacent tank and causing serious hazard.

VOCs fire and explosion cause not only a loss of liquid cargo, but also a damage of the tank structure, air pollution, marine pollution, casualties, and even the sinking of the ship.

\section{Risk hazards of collision and grounding in the liquid cargo tank}

In the process of navigation, a collision takes place in the oil tanker, causing some hazards or losses, especially vertical collision occurs between two ships, the hazards are the greatest. When vertical collision occurs, leading to structure damages and oil leakages in oil tank, even a fire, casualties and sink of the ship. When a grounding occurs, it will lead to structure damages and oil leakages in liquid cargo tank and marine pollution etc..

\section{Risk hazards of marine oilspill}

In oil loading and unloading processes, oil pipeline may burst, causing a oilspill when the pressure of the cargo oil rises rapidly, or when oil pipeline is damaged or aged.

In the navigation process of oil tanker loading lots of cargo oil, an oilspill may take place owing to the collision, grounding, damaged or aged structures of the oil tank.

Oilspill will not only lead to a loss of cargo oil, but also cause harm to the marine resources of the birds, fishes and shrimps, seaweeds, shellfishes etc. , affecting their ability to survive, and even leading to death.

Marine oilspill yet is hazardous to the seashore and coastal natural environment hazards, reducing its values of the entertainment, recuperation, scouring bath etc. , deteriorating natural landscapes.

\section{RESUlts}

In the paper, methods of VOCs processing were summarized, and the current method of VOCs processing in 
the sloshing liquid cargo tank and its problems or deficiencies were analyzed. To tackle problems or deficiencies in the current method of VOCs processing in the sloshing liquid cargo tank, the system diagram of the sloshing liquid cargo tank including 13 basic elements was established based on the theory of MMEMSE, and its functions of every element in the sloshing liquid cargo tank system was analyzed. The main risks of escaping and diffusion of the gas mixtures, VOCs fires and explosions, collisions and groundings, and marine oilspills etc. in the sloshing liquid cargo tank in oil tanker were concluded from operation processes of loading, unloading and COW in the sloshing liquid cargo tank system. What's more, the formation process of risks and their hazards were analyzed too

\section{CONCLUSIONS}

The potential risks of the sloshing liquid cargo tank in oil tanker are inevitable, but the risks can be controlled. With the effective implementation of control measures, ship accidents can be avoided to the most degree. Analysis on risks and their hazards of the sloshing liquid cargo tank in oil tanker is of a previous research, further studies are as follows, risk assessment, risk control, risk evolution, model experiments, simulation analysis and structure optimization of the sloshing liquid cargo tank etc.

\section{ACKNOWLEDGMENT}

The present research was partly funded by Zhejiang Provincial Natural Science Foundation of China, No. LQ14E090001, nation natural foundation of China, No. 51079129 and Zhejiang Provincial department of education project, No. Y201328406. Also, the research was supported by ZheJiang Ocean University, thanks Lu Jin-shu, Li Yu-le, Gan Xue-zhen for their selfless and sincere support.

\section{REFERENCES}

[1] Wang Yun-long, Jin Chao-guang, Ji Zhuo-shang etc. Evolution and trends of hull structure design of the oil tanker[J]. Chinese journal of ship research, 2011, 01: 1-6.

[2] ITOPF.Oil tanker spill statistics 2014 [EB/OL]. [2015-0204].http://www.itopf.com/knowledge-resources/data- statistics/ statistics.

[3] Fire prevention of China online. Studies on the characteristics and measures of the fires in the cargo oil tank[EB/OL]. [2015-0204].http://119.china.com.cn/ txt/ 2008-01/24/content_2031592.htm,

[4] Zhu Zhe-ye.Study on Sloshing Effect of Mass Transfer between Vapor and Liquid Phases in Oil Tank of Fishing Boats[D]. Zhoushan, Zhejiang Ocean University,2014.

[5] Liu Jing, Li Zi-li, Sun Yun-feng et al. Research Progress in Oil Vapor Recovery Technology Worldwide [J]. Oil \& Gas storage and transportation,2010,29(10):726-729.

[6] Li Cheng, Li Jun-ming. Economic analysis of light hydrocarbons recovering with condensation method [J]. Oil \& Gas storage and transportation,2013,32(11):1206-1208.

[7] Miao Zhi-hua, Wang Meng et al. Technology and application of condensing oil vapor recovery[J]. Cryo. \& Supercond, 2011,39(06):48-52.

[8] Zhang Xiang-ping, Liu Jie-bo.Absorption method and adsorption method combining applied to oil and gas recovery[J]. Environmental protection in peterochemical industry, 2006, 29(03):57-61.

[9] Kong Xiang-qiang, Cao Dong-hui, Yang Qian-ming et al. Optinal operation of gasoline vapor recovery equipment by condensation [J]. Energy Engineering, 2008,05:59-63.

[10] Bu Chang-sen, Cheng Wei-min, Zhou Gang et al. Studies on Safety analysis and assessment of Man-Machine-Environment System Engineering[J]. Industry safety and environmental protection,2009, 35(02):44-46.

[11] Chinese Maritime Service Center. Dalian, The convention supplements of training certificate in oil tanker and chemicals ship[M]. Dalian, Dalian maritime university press, 2012.

[12] Hu Shen-ping, Fang Quan-gen, Xia Hai-bo. Study on formal safety assessment on ship's navigation and modeling based on relative risk[J]. Journal of Dalian Maritime University, 2005, 31(02):18-22. 